\title{
MergeReference: A Tool for Merging Reference Panels for HLA Imputation
}

\author{
Seungho Cook, Buhm Han* \\ Department of Convergence Medicine, University of Ulsan College of Medicine and Asan Institute for Life Sciences, \\ Asan Medical Center, Seoul 05505, Korea
}

\begin{abstract}
Recently developed computational methods allow the imputation of human leukocyte antigen (HLA) genes using intergenic single nucleotide polymorphism markers. To improve the imputation accuracy in HLA imputation, it is essential to increase the sample size and the diversity of alleles in the reference panel. Our software, MergeReference, helps achieve this goal by providing a streamlined pipeline for combining multiple reference panels into one.
\end{abstract}

Keywords: human leukocyte antigens, major histocompatibility complex

Availability: MergeReference is publicly available at http://software.buhmhan.com/MergeReference.

\section{Introduction}

Human leukocyte antigen (HLA) in the major histocompatibility complex (MHC) region is an important genetic factor for a wide range of human diseases, such as type 1 diabetes [1] and rheumatoid arthritis [2, 3]. In association studies of HLA, it is essential to investigate the high-resolution genotypes of HLA genes. However, genotyping HLA alleles in a large number of samples can be prohibitively expensive due to the high HLA typing cost. To overcome this challenge, imputation approaches have been recently developed to impute HLA alleles from intergenic single nucleotide polymorphism (SNP) data [4-6]. These approaches, of which SNP2HLA [4] is widely used, can impute high-resolution HLA alleles efficiently and have been utilized in a number of large-scale fine-mapping HLA analyses of diseases [1-3, 7, 8].

Although the use of imputation approaches has become prevalent, imputation errors can always be present. To reduce imputation errors, it is often necessary to use a large reference panel that can capture diverse HLA alleles. Both the size of the panel and the diversity of alleles can increase if we can use multiple reference panels at the same time. However, in current HLA imputation frameworks, there is no streamlined pipeline for simultaneously utilizing multiple reference panels.

In this study, we present a software package, MergeReference, that merges multiple reference panels in SNP2HLA [4] format into a single panel for HLA imputation. By merging multiple reference panels of similar ethnicities, we can increase both the sample size and the allele diversity of the panel and therefore improve the imputation accuracy. We compared the performance of the Pan-Asian reference panel [9], the Korean reference panel [10], and the merged reference panel generated by our software by masking and imputing the HLA alleles in the HapMap [11] Asian dataset. The merged panel achieved an average 4-digit accuracy of six HLA genes of $93.9 \%$, whereas the Pan-Asian and the Korean panels had $86.6 \%$ and $91.5 \%$ accuracy rates, respectively, demonstrating that the simultaneous use of multiple reference panels improves the accuracy. MergeReference is freely available at http://software.buhmhan.com/MergeReference.

\section{Methods \\ MergeReference}

MergeReference is a software package that merges multiple reference panels for HLA imputation. We assume that the panels are in SNP2HLA format [4]. MergeReference consists 
of the following four steps (Fig. 1). Although these steps are for merging two panels, merging more than two panels can be done straightforwardly by repeatedly applying the same procedure to pairs of panels until all panels are merged into one.

\section{Step 1. Extracting SNP and HLA allele data}

Given two reference panels to be merged, we extract SNP data from them using PLINK [12]. Then, we extract HLA genotype information from the reference panel. Because each allele in an HLA gene is coded as a binary marker in SNP2HLA format, extracting HLA allele information requires the examination of the entire set of binary markers for an HLA gene. We use our Python script to perform this extraction.

\section{Step 2. Combining SNP data}

In this step, we combine the SNP data of the two panels. First, we select overlapping SNPs that exist in both panels. An alternative approach would be to keep all SNPs as partially missing data. However, in this alternative approach, the missing values in this step will be automatically imputed in step 4 and will be used as reference data in the imputation. Because using imputed data as reference data can degrade the performance, we avoided this alternative approach. Second, we check and correct the strand flips of the SNPs between the two panels. For A/T and G/C SNPs with strand flips that are difficult to detect, we use frequency information.

\section{Step 3. Combining HLA data}

We combine the HLA data of the two panels. As we did in step 2 , in order to avoid using imputed values as reference data, we only keep overlapping HLA genes that exist in both panels.

\section{Step 4. Creating a merged reference panel}

Given the combined SNP data from step 2 and the combined HLA data from step 3, we employ MakeReference software in the SNP2HLA package [4] to construct the new merged reference panel in SNP2HLA format.

\section{Results}

\section{Merging Korean and Pan-Asian panels}

To test our MergeReference software, we merged the Pan-Asian panel $(n=441)$ [9] and the Korean panel $(n=413)$ [10]. The Korean panel included 4526 SNPs, and the Pan-Asian panel included 4,603 SNPs in the MHC region (chr6:25-34Mb). The Korean panel had genotype information on six HLA genes (A, B, C, DRB1, DPB1, and DQB1), and the Pan-Asian panel had genotype information on eight

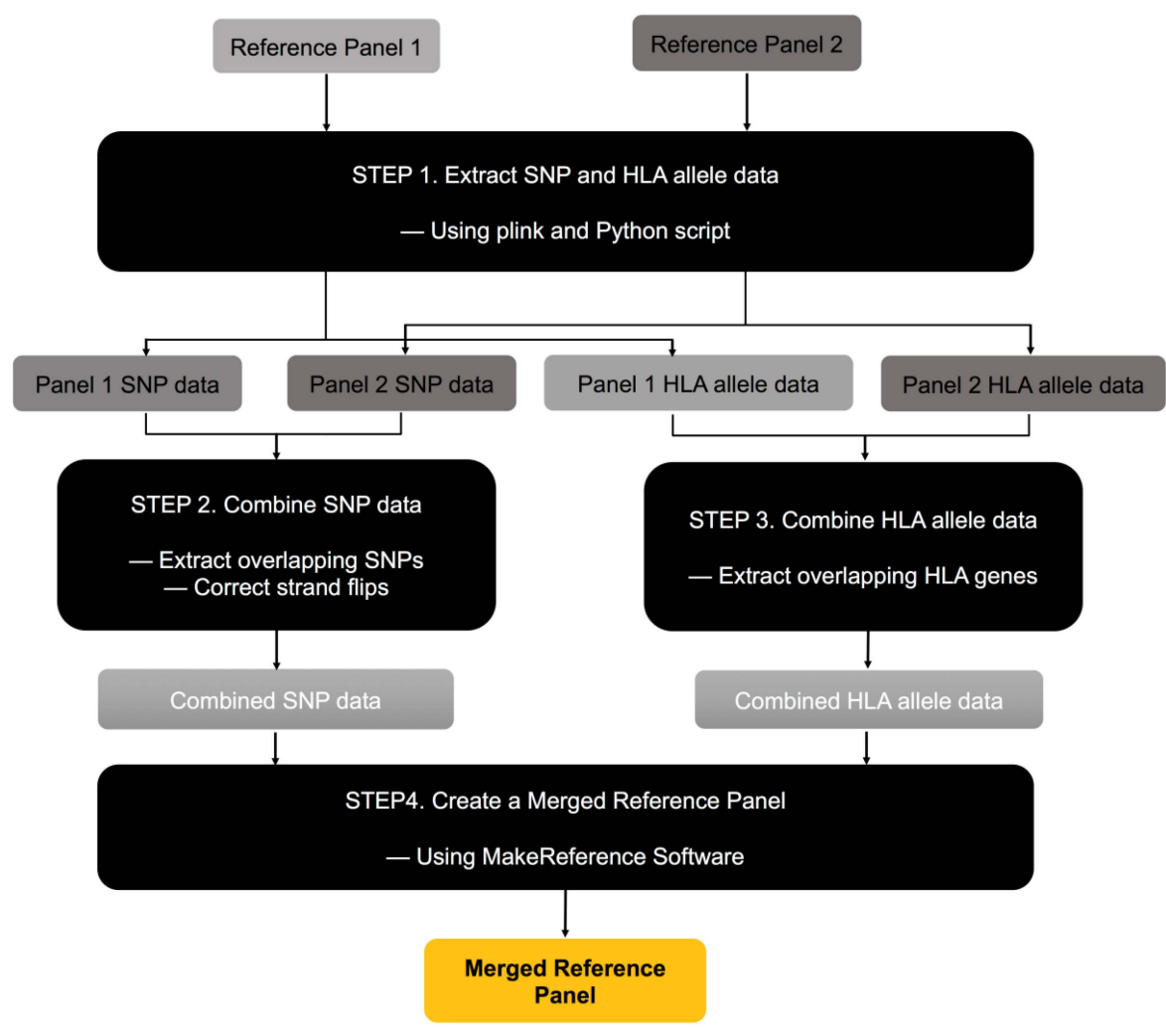

Fig. 1. The four steps of MergeReference for merging two panels into one. SNP, single nucleotide polymorphism; HLA, human leukocyte antigen. 
HLA genes (A, B, C, DRB1, DPA1, DPB1, DQA1, and DQB1). After applying MergeReference, 2,995 overlapping SNPs and the genotype information of six overlapping HLA genes were left. Merging the two panels took less than 10 min on a standard computer $(2.7 \mathrm{GHz}$ Intel Core i5 CPU, 8 GB memory).

\section{Merged panel can improve performance}

To compare the imputation accuracy of different panels, we used the 61 HapMap Asian samples from Beijing, China (CHB) and Tokyo, Japan (JPT) as benchmark data [11]. We masked the HLA information of these individuals, imputed them, and compared the imputed genotypes to the true genotypes. We evaluated three panels: the Pan-Asian panel, the Korean panel, and the merged panel of the two. The merged panel achieved an average 4-digit accuracy of six HLA genes of $93.8 \%$, whereas the Pan-Asian and Korean panels had $85.4 \%$ and $91.5 \%$ accuracy rates, respectively (Fig. 2). The per-HLA gene accuracies are described in

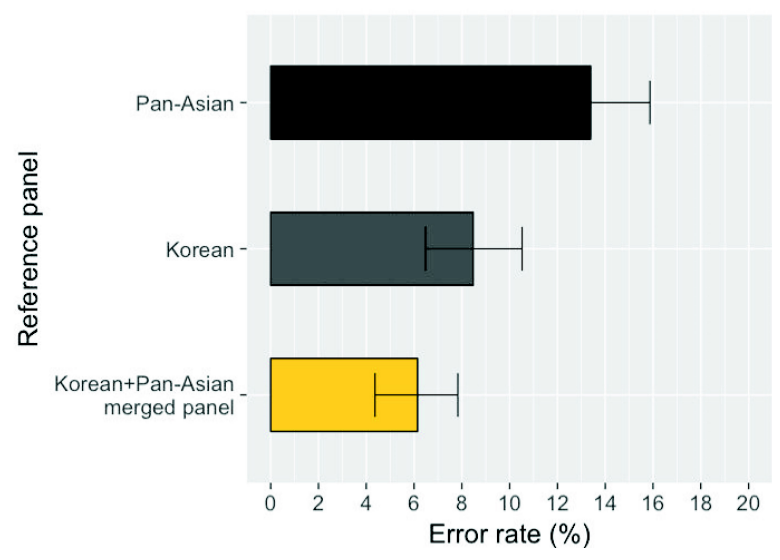

Fig. 2. Error rates in imputing HLA information on 61 HapMap CHB +JPT samples. We evaluated error rates using three different reference panels. The error rates were based on 4-digit imputation using SNP2HLA and were averaged over six HLA genes (A, B, C, DRB1, DPB1, and DQB1). The bars indicate 95\% confidence intervals. CHB, Beijing, China; JPT, Tokyo, Japan; HLA, human leukocyte antigen.
Table 1. The performance of the merged panel was equal or superior to that of the individual panels for all HLA genes. The improvement in performance was notable for HLA-DRB1 (Pan-Asian, 85.2\%; Korean, 89.3\%; merged, 95.1\%).

\section{Discussion}

We have developed MergeReference, a software package that provides a streamlined pipeline for combining multiple reference panels into one for HLA imputation. In a previous study, Kim et al. [13] also evaluated the gain in performance of merging reference panels for HLA imputation, although they did not provide a software pipeline. Similar to our work, their results supported the utility of merging panels, particularly when the ethnicities are similar. However, merging Asian panels with a European panel did not provide better performance for imputing HLA in Asian samples [13]. Therefore, determining whether merging multiple panels increases the accuracy depends on how similar the ethnicities of the panels are. If it is unclear whether merging panels would help or not, it can be good practice to evaluate the approximate accuracy by using a public dataset, such as HapMap, or setting aside a subset of the panel samples as a test dataset. Another issue that can affect the performance of the merged panel is the sample size balance. We found that if one panel is much larger (e.g., by 10-fold) than the other panel, the merged panel often has essentially the same performance as the single large panel. In such situations, subsampling from the large panel may improve the balance of the two panels, despite the decreased sample size. Further investigations will be needed to thoroughly explore these issues related to the gain in performance with merging, and we expect that our software pipeline will facilitate such explorations in future studies.

\section{Acknowledgments}

This work was supported by a National Research Foundation of Korea (NRF) grant, funded by the Korean gover-

Table 1. Per-HLA gene imputation accuracy in imputing HLA information on 61 HapMap CHB + JPT samples

\begin{tabular}{lccccccc}
\hline \multicolumn{1}{c}{ HLA gene } & $\mathrm{A}$ & $\mathrm{B}$ & $\mathrm{C}$ & $\mathrm{DRB} 1$ & $\mathrm{DPB} 1$ & DQB1 & Average \\
\hline Pan-Asian & 0.893 & 0.721 & 0.893 & 0.852 & 0.926 & 0.909 & 0.866 \\
& $(0.838-0.948)$ & $(0.641-0.801)$ & $(0.891-0.895)$ & $(0.838-0.948)$ & $(0.880-0.972)$ & $(0.858-0.960)$ & $(0.841-0.891)$ \\
Korean & 0.901 & 0.893 & 0.983 & 0.893 & 0.926 & 0.893 & 0.915 \\
& $(0.848-0.954)$ & $(0.838-0.948)$ & $(0.960-1.001)$ & $(0.838-0.948)$ & $(0.876-0.972)$ & $(0.838-0.948)$ & $(0.895-0.935)$ \\
Merged panel & 0.943 & 0.910 & 0.992 & 0.951 & 0.926 & 0.910 & 0.939 \\
& $(0.902-0.984)$ & $(0.859-0.961)$ & $(0.976-1.001)$ & $(0.913-0.990)$ & $(0.876-0.972)$ & $(0.859-0.961)$ & $(0.922-0.956)$ \\
\hline
\end{tabular}

The accuracies and $95 \%$ confidence intervals were based on 4-digit imputation using SNP2HLA

HLA, human leukocyte antigen; CHB, Beijing, China; JPT, Tokyo, Japan. 
nment (MSIP) [grant number 2016R1C1B2013126].

\section{Authors' contribution}

Conceptualization: $\mathrm{BH}$

Formal analysis: SC

Funding acquisition: $\mathrm{BH}$

Writing - original draft: $\mathrm{SC}, \mathrm{BH}$

Writing - review \& editing: SC, BH

\section{References}

1. Hu X, Deutsch AJ, Lenz TL, Onengut-Gumuscu S, Han B, Chen WM, et al. Additive and interaction effects at three amino acid positions in HLA-DQ and HLA-DR molecules drive type 1 diabetes risk. Nat Genet 2015;47:898-905.

2. Raychaudhuri S, Sandor C, Stahl EA, Freudenberg J, Lee HS, Jia $\mathrm{X}$, et al. Five amino acids in three HLA proteins explain most of the association between MHC and seropositive rheumatoid arthritis. Nat Genet 2012;44:291-296.

3. Han B, Diogo D, Eyre S, Kallberg H, Zhernakova A, Bowes J, et al. Fine mapping seronegative and seropositive rheumatoid arthritis to shared and distinct HLA alleles by adjusting for the effects of heterogeneity. Am J Hum Genet 2014;94:522-532.

4. Jia X, Han B, Onengut-Gumuscu S, Chen WM, Concannon PJ, Rich SS, et al. Imputing amino acid polymorphisms in human leukocyte antigens. PLoS One 2013;8:e64683.

5. Dilthey AT, Moutsianas L, Leslie S, McVean G. HLA*IMP: an integrated framework for imputing classical HLA alleles from SNP genotypes. Bioinformatics 2011;27:968-972.

6. Zheng X, Shen J, Cox C, Wakefield JC, Ehm MG, Nelson MR, et al. HIBAG-HLA genotype imputation with attribute bagging. Pharmacogenomics J 2014;14:192-200.

7. Dunstan SJ, Hue NT, Han B, Li Z, Tram TT, Sim KS, et al. Variation at HLA-DRB1 is associated with resistance to enteric fever. Nat Genet 2014;46:1333-1336.

8. Okada Y, Han B, Tsoi LC, Stuart PE, Ellinghaus E, Tejasvi T, et al. Fine mapping major histocompatibility complex associations in psoriasis and its clinical subtypes. Am J Hum Genet 2014;95:162-172.

9. Pillai NE, Okada Y, Saw WY, Ong RT, Wang X, Tantoso E, et al. Predicting HLA alleles from high-resolution SNP data in three Southeast Asian populations. Hum Mol Genet 2014;23: 4443-4451.

10. Kim K, Bang SY, Lee HS, Bae SC. Construction and application of a Korean reference panel for imputing classical alleles and amino acids of human leukocyte antigen genes. PLoS One 2014;9:e112546.

11. International HapMap Consortium. The International HapMap Project. Nature 2003;426:789-796.

12. Purcell S, Neale B, Todd-Brown K, Thomas L, Ferreira MA, Bender D, et al. PLINK: a tool set for whole-genome association and population-based linkage analyses. Am J Hum Genet 2007;81:559-575.

13. Kim K, Bang SY, Lee HS, Okada Y, Han B, Saw WY, et al. The HLA-DR $\beta 1$ amino acid positions 11-13-26 explain the majority of SLE-MHC associations. Nat Commun 2014;5:5902. 